[Contribution from the Departmeint of Chemistry, Hahnemann Medicat ColLEGE OF CHTCAGO.]

[In affiliation with Valparaiso University.]

\title{
THE INDIVIDUALITY OF ERYTHRODEXTRIN.
}

BY J. C. BLAKE.

Received Oct. 11, 1920.

In $1916^{1}$ the author arrived at the provisional conclusion that fractional precipitation of $1 \%$ solutions of dextrins probably yielded pure erythrodextrin as the portion thrown down by 50 to $65 \%$ alcohol (by volume). Later $^{2}$ it was found that the separation from achro-odextrin became incomplete if the concentration of the dextrin solution was greatly increased, so that the method was more or less impractical. Since that time somewhat extended efforts have been made to prepare and isolate pure erythrodextrin for the purpose of standardizing amylolytic activity and facilitating amylolytic determinations. Without attempting to review all this work in detail, it may be recorded that such dextrins or British gums were found to be preferable as sources of erythrodextrin that give deep red colors with excess of iodine-water, preceded by little or no blue coloration. If much of the blue-producing dextrin (amylodextrin) is present low concentrations of alcohol will remove it completely from part of the erythrodextrin, but probably half of the latter ingredient is lost in the precipitate. In dextrin solutions from which fragments of the cell walls have been removed by filtration this precipitate takes the form of sphero-crystals, in which the erythrodextrin seems to be present in solid solution. ${ }^{3}$

It was thought for some time that after all of the amylodextrin had been removed from approximately 20\% solutions in the manner just indicated, all of the higher polysaccharicles had also been removed. Never. theless it was observed in numerous cases that further addition of alcohol, instead of yielding the heavy liquid in which form erythrodextrin subsequently precipitates, continued to yield small crops of sphero-crystals, thus indicating that part of the higher polysaccharides ${ }^{4}$ was still present. This conclusion was confirmed by examination of the "erythrodextrin" precipitated by slight further addition of alcohol. "The heavy liquids thus precipitated from the same concentration of different dextrins by the same precentage of alcohol had widely varying powers of turning red with iodine and many of them became turbid on standing, and became largely insoluble in cold water.

i THIS JOURNAL, 38 , 125 I (r916).

2 Ibid., 39, 316 (1917).

${ }^{3}$ Blake, ibid., 40, 635 (ror 8 ).

4 The substance mainly responsible for these crystals is thought to be the same as that forming the cell walls of raw starch; hence the name "artificial starch." This substance is probably amylocellulose 
In order to arrive at any definite conclusion with regard to the relative purity of the erythrodextrin in such preparations it was necessary to determine their solid content. Heating to roo $^{\circ}$ slowly decomposes the erythrodextrin. Further addition of alcohol changes the heavy liquid to a coherent and adherent gum, which seemed even harder to work with than the heavy liquid. Hence 4 such liquids ${ }^{1}$ were desiccated over calcium chloride, the resulting solid being powdered as soon as it became

1 Dextrin (British gum) No. I was prepared by heating wheat starch for 65 days at $100^{\circ}$, except that the temperature reached $140^{\circ}$ the sixth day. The amylodextrin and higher polysaccharides were precipitated by $30 \%$ alcohol from a $7.3 \%$ solution (final concentration). The erythrodextrin was precipitated as a heavy liquid by $50 \%$ alcohol. This liquid was used in Tabie I. Further addition of alcohol to the supernatant liquid gave a white solid precipitate by local excess, which quickly redissolved on stirring (achro-odextrin).

Dextrin No. 2 was from a white dextrin kindly furnished for this purpose by the Corn Products Refining Co. Two hundred g. of this material was extracted with $74 \%$ alcohol by means of a percolator for 40 days, by the end of which time the extract had reached the constant polarization of 0.54 (glucosimeter degrees), due chiefly to erythrodextrin and amylocellulose (sugars and achro-odextrin having been present in the early extracts). The presence of amylocellulose in the extract from the first was nicely shown by the formation of "artificial starch" on adding ether. As the ether evaporated, the sphero-crystals redissolved. The residue from the $74 \%$ alcoholic extract was then leached in a similar manner with $42 \%$ alcohol. The first extract with this solvent gave a slight deposit on standing of equal portions of cell fragments and sphero-crystals, together with a few long colorless needles. (Cf. Blake, THis Journal, 4o, 636 ( $19 \times 8)$.) Extracts No. 2 to 8 of this series contained the most erythrodextrin. From a mixture of these extracts a slight precipitate of amylodextrin and higher polysaccharides was obtained by making the alcoholic content $55 \%$. From the filtrate a gummy precipitate containing most of the erythrodextrin was obtained by increasing the alcoholic content to $75 \%$ (used in Table I). The supernatant liquid contained a small amount of achro-odextrin.

Dextrin. No. 3 was obtained from a mixture of dextrins made by heating corn starch with acid until about $99 \%$ of the material was soluble in cold water. Two hundred $\mathrm{g}$. of this material was extracted with $74 \%$ alcohol in the manner just described, a constant polarization of 0.80 being reached after 29 days. Of the subsequent extracts with 42 to $50 \%$ alcohol, Nos. 4 to 9 contained the purest erythrodextrin. From a mixture of those extracts a slight precipitate of amylodextrin, higher polysaccharides and some erythrodextrin was obtained by increasing the alcoholic content to $50 \%$. From the filtrate a heavy liquid, containing most of the erythrodextrin, was precipitated by increasing the alcoholic content to $72 \%$ (used in Table I). This heavy liquid contained much erythramylum when tested by the method heretofore described (THIS JOURNAL, $40,623)$; that is, it gave much red color with little iodine-water before any of the blue color due to amylodextrin appeared. Dextrin No. 4 was from a yellow commercial dextrit rich in erythrodextrin, cell walls insoluble. This dextrin had stood in $10 \%$ solution for 2 years, without preservative and without apparent change. Alcohol added to the supernatant liquid up to $44 \%$ gave the usual precipitate, containing all but a trace of the amylodextrin. From the filtrate the heavy liquid used in Table I was precipitated by increasing the alcoholic content to $59 \%$.

Dextrin No. 5 (infra) was obtained by mixing together several such heavy liquids as those described under dextrins Nos. 2 to 4 , obtained from corn starch. 
brittle. The weight of Dextrin No. I becarne constant after 6 months; Dextrins Nos. 2 to 4 were still slowly losing weight after one year, at which time the subsequent determinations were made.

Table I contains a summary of the early procedures carried out with these dextrins.

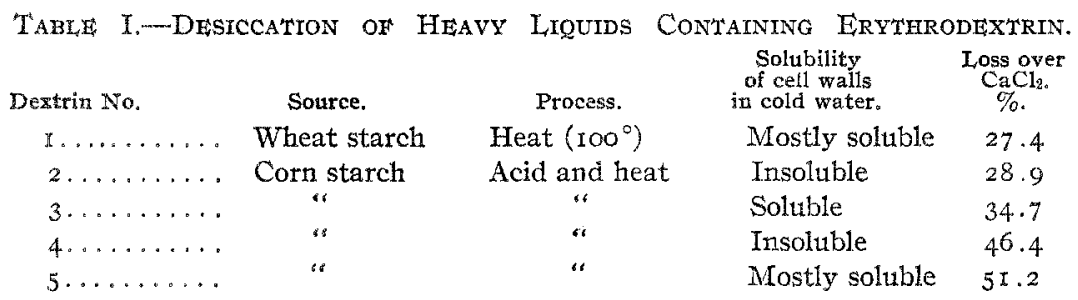

In order to determine the nature of these dried dextrins, solutions were made containing $5 \mathrm{~g}$. of the dried material per roo cc. These solutions were then polarized in a 2 -dcm. tube at $25^{\circ}$ on a glucosimeter, ${ }^{1}$ and their power of coloring red with iodine was determined, as likewise their digestibility to the achromic point with fresh $1 / 18$ strength saliva (final concentration), preserved overnight with toluene, and their power of reducing Benedict's quantitative reagent. Table II contains the data thus obtained.

\begin{tabular}{|c|c|c|c|c|c|}
\hline Dextrin No. & $\begin{array}{l}\text { Polarization } \\
\text { on } \\
\text { glucosimeter. }\end{array}$ & $\begin{array}{l}\text { Red with iodine for } \\
\text { one cc. (corrected), } \\
\text { Lovibond scale } \\
=d \text {. }\end{array}$ & $\begin{array}{c}\text { Time } \\
\text { to achromic } \\
\text { point with } \\
\text { 1/18 strength } \\
\text { saliva in minutes } \\
=t .\end{array}$ & $d / t_{0}$ & $\begin{array}{l}\text { "Glucosse by } \\
\text { Benedict's } \\
\text { reagent. } \\
\% \%\end{array}$ \\
\hline$x \ldots \ldots$ & +16.58 & 53 & 3.00 & 18 & 2.0 \\
\hline $2 \ldots \ldots$ & +16.00 & 37 & I. 97 & I9 & 1.9 \\
\hline $3 \ldots . .$. & +15.60 & 35 & 1.72 & 20 & I. 4 \\
\hline $4 \ldots .$. & +15.42 & 33 & I. 92 & I7 & 2.0 \\
\hline $5 \ldots \ldots \ldots$ & +16.20 & 40 & 2.25 & I8 & 1. 6 \\
\hline
\end{tabular}

Accepting these results, momentarily, at their face value one would conclude that these products each contained about $2 \%$ sugar. But the original raw dextrins gave only a faint test with Benedict's qualitative reagent and the method of separating the heavy liquids from which these products were obtained precluded the presence of sugar. Hence it seems probable that this uniform copper reduction measures the amount of decomposition of erythrodextrin during the heating necessary to make the test, amounting to $2.0 \%$ for the purest erythrodextrin obtained (No. I).

The other results require further elucidation. Assuming that the depth of the red color given with iodine-water measures the amount of erythrodextrin present, and that the time required for digestion to the achromic

I In all cases tested sodium light gave the same reading as incandescent light. No mutarotation was observed. This instrumant reads percentages of glucose when a. $200 \mathrm{~mm}$. tube is used. 
point measures the same quantity, ${ }^{1}$ it is evident that the ratio of these 2 determinations ought to be constant. The values thus obtained are given in Column 5 of Table II, and are probably constant within the experimental error.

In order to establish the last conclusion more firmly and to illustrate the present use of the author's method ${ }^{2}$ of determining the depth of the red color given by erythrodextrin with iodine, the details of such determinations, whereby the values given in Table II were arrived at, are here recorded.

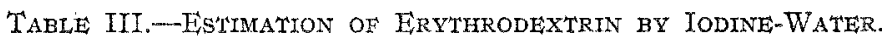

One cc. of $5 \%$ dextrin solution in cylinder of Duboseq colorimeter of $6.55 \mathrm{sq} . \mathrm{cm}$. cross section.

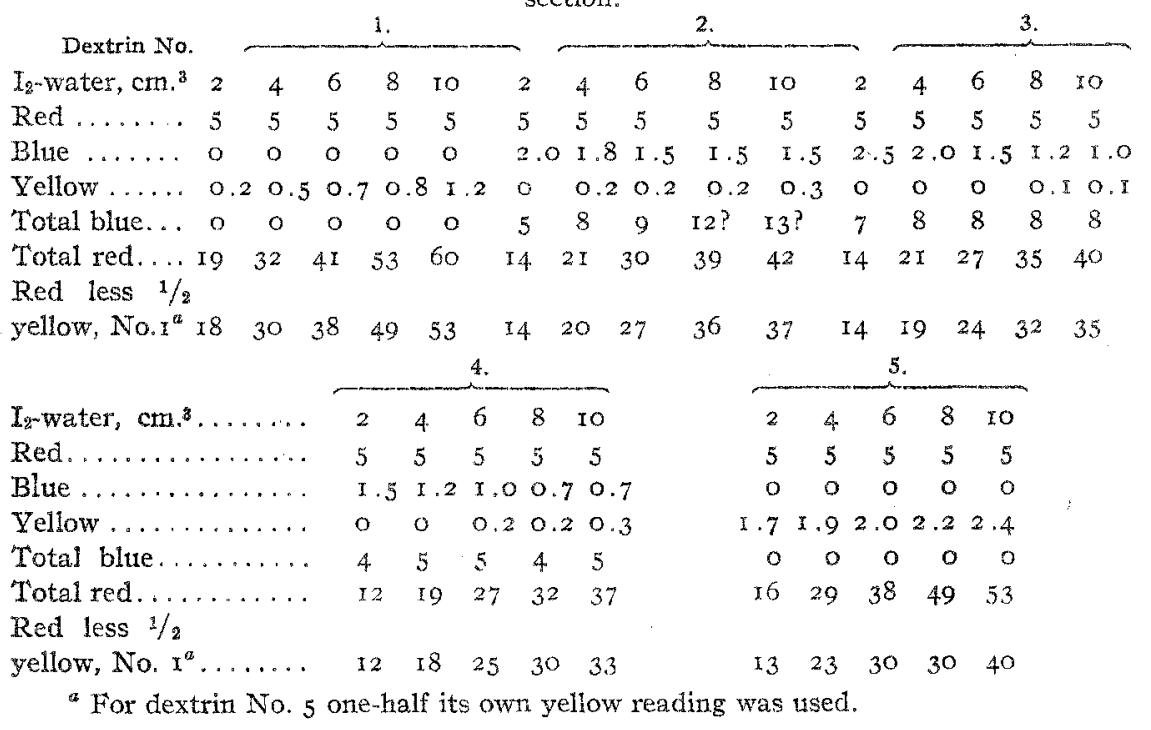

It is well known that the depth and the tone of the color given by iodine with dextrin solutions vary with the proportion of iodine added. In $1918^{3}$ the author indicated that the depth of color produced by iodine with erythrodextrin reaches a maximum when the iodine is added in slight excess, that the presence of amylodextrin is not a disturbing factor, and that the red color due to excess of iodine can be corrected for by subtracting from the total red color observed $40 \%$ of the total yellow color observed. It has since been found that no advantage is gained in adding more iodine after its yellow tint becomes distinctly discernible when amylodextrin is present, owing to the fact that the blue iodide nettralizes the yellow color of iodine until considerable excess of the latter is present.

I Blake, ThIs Journal, 39, 318 (I9I7), Table III.

2 ThIS JOURNAL, 40,623 (1918).

Ibid., 40,624-26 (1918). 
It has likewise been found that the red color of iodine-water equals $50 \%$ of its yellow color (expressed on the Lovibond scale), instead of $40 \%$ as previously recorded (Table IV).

Table IV.-Lovibond Glasses Matching Different Thicknesses of IodingWATER.

Red glasse
0.2
0.6
2.5
3.5

Yellow glasses.
$\begin{gathered}0.5 \\ 1.0 \\ 5.0 \\ 7.2\end{gathered}$

From the next to the last line of Table III it will be noticed that the total red color steadily increases with increase of iodine-water. The last line of the table contains the same results lessened by $1 / 2$ the yellow color observed with Dextrin No. I. It will be noticed that the values become nearly constant with the final increases in the proportion of iodine-water. The last value, that obtained with Io volumes of iodine-water, probably yields for solutions of this strength about the best result obtainable for comparative purposes, since further addition of iodine introduces greater uncertainties in correcting for its red color. This correction for Dextrins Nos. 2 to 4 was made in accordance with the yellow color developed in Dextrin No. I because this dextrin was entirely free from amylodextrin, whereas in the case of Dextrins Nos. 2 to 4 the blue color of the iodide of the small amount of amylodextrin present largely neutralized the yellow color due to the excess of iodine, without apparently affecting its red color.

It is plain from the foregoing results that Dextrin No. I was far richer than any of the others in erythrodextrin. Its specific rotation at $25^{\circ}$, 174 , agrees well with the value ( 170 ) heretofore obtained ${ }^{1}$ by fractionation of a commercial sample of dextrin "purified by alcohol."

The chief impurity in Dextrins Nos. 2 to 4 was thought to be amylocellulose. It was found that most of the erythrodextrin could be precipitated by ammonium sulfate from the heavy liquids heretofore described, other substances remaining in the supernatant liquid. Accordingly $45^{\circ} \mathrm{cc}$. of heavy liquid, resembling those finally used in Nos. 2 to 4, was treated with $197 \mathrm{~g}$. of solid ammonium sulfate. Two liquid layers resulted, the heavier one, consisting of $177 \mathrm{cc}$., containing nearly all the ery throdextrin. The supernatant liquid contained a considerable amount of other organic material, precipitable, along with ammonium sulfate, by the further addition of alcohol. The heavy liquid just described was freed from ammonium sulfate by heating to $60^{\circ}$ with a slight excess of barium carbonate for 2 hours. From the filtrate thus obtained the erythrodextrin was again precipitated as a heavy liquid by $42 \%$ alcohol. This liquid (Table I, No. 5) dried to a solid over calcium chloride in 2

: This JoUrnar, 39, $320(19 \times 7)$. 
days, and its weight became constant after 48 days, thus showing that the substance which held water so tenaciously in Dextrins Nos. 2 to 4 had been removed by the treatment with ammonium sulfate.

The properties of the material thus obtained are included in Tables II and III, the digestion having been made with the same sample of saliva used with the other dextrins. That saliva preserved with toluene is stable for years was announced by the author at the Cleveland meeting of the American Chemical Society (1918), and independently soon thereafter by Myers and Scott. ${ }^{1}$

It will be noticed from Table III that the last traces of amylodextrin have been removed and that the ratio of the red color with iodine to the time required for digestion to the achromatic point, 18 , is the same as that obtained for Dextrin No. $\mathrm{r}$, which this dextrin most closely resembles. But the red color and the time required for digestion are only $75 \%$ of the values given by Dextrin No. $I$; that is to say, Dextrin No. 5 contained only $75 \%$ as much erythrodextrin as Dextrin No. I. This loss of erythrodextrin must be attributed to a partial conversion to achro-odextrin during the heating with barium carbonate. The lessened content of erythrodextrin also accounts for the smaller ratio of red to yellow observed on adding the same proportion of iodine-water (Table III).

With this evidence of the individuality of erythrodextrin the author's efforts in this direction will be necessarily terminated, at least temporarily; but erythrodextrin nearly free from amylodextrin will continue in extensive use in this laboratory as an accurate and ready means of determining relative amylolytic activity.

CHFCAOO, Il,

[CONTRibution from the Pharmacology Departiment, Untuersity of Minnesota. ]

\section{MERCURY COMPOUNDS OF SOME PHENYL CARBINOLS. ${ }^{2}$}

\section{By Merrigi, C. Har't and Arthur D. Htrschifiderr.} Received October 21, 1920.

In spite of the large number of organic mercury compounds which have hitherto been prepared $^{3}$ we have been unable to find references to any compounds of mercury and arsenic with phenyl carbinols.

In view of the relatively low toxicity of phenyl carbinols, the presence of carbinol groups in many of the most active natural alkaloids and the interesting local anesthetic and anti-spasmodic properties possessed by

' Myers and Scott, Thrs Journal, 40, I713 (I918).

2 This work was done with the aid of funds granted by the United States Interdepartmental Social Hygiene Board, for the investigation of the antiseptic and chemotherapeutic action of phenolic alcohols and their derivatives upon the gonococcus and the spirochaete.

a F. C. Whitmore, I. Ind. Eng. Chem., I I, 1083 (x9r9). 